\title{
Quark disconnected diagrams in chiral perturbation theory
}

\author{
Michele Della Morte ${ }^{a}$ and Andreas Jüttner ${ }^{b}$ \\ ${ }^{a}$ Institut für Kernphysik, Johannes Gutenberg-Universität Mainz, \\ Johann-Joachim-Becher Weg 45, D-55099 Mainz, Germany \\ ${ }^{b}$ CERN, Physics Department, TH Unit, \\ CH-1211 Geneva 23, Switzerland \\ E-mail: morte@kph.uni-mainz.de, juettner@kph.uni-mainz.de
}

ABSTRACT: We show how quark-disconnected and quark-connected contributions to hadronic $n$-point functions can be written as independent correlators for which one can derive expressions in partially quenched chiral effective theory. As an example we apply the idea to the case of the hadronic vacuum polarisation. In particular, we consider the cases of the $N_{f}=2$ theory without and with a partially quenched strange quark and also the $N_{f}=2+1$ theory. In the latter two cases a parameter-free prediction for the disconnected contribution at NLO in the effective theory is given. Finally we show how twisted boundary conditions can then be used in lattice QCD to improve the $q^{2}$ resolution in the connected contributions even when flavour singlet operators are considered.

Keywords: Lattice QCD, Nonperturbative Effects, Electromagnetic Processes and Properties, Chiral Lagrangians

ARXIV EPRINT: 1009.3783 


\section{Contents}

$\begin{array}{llr}1 & \text { Introduction } & 1\end{array}$

2 General argument $\quad 2$

3 Hadronic vacuum polarisation 4

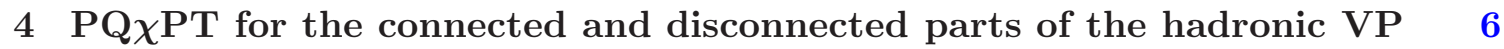

$4.1 O\left(p^{2}\right)$-Lagrangian $\quad 6$

$\begin{array}{lll}4.2 & O\left(p^{4}\right) \text {-Lagrangian } & 7\end{array}$

$\begin{array}{lll}4.3 & \text { Contributing diagrams } & 7\end{array}$

5 Results and applications to lattice QCD $\quad 8$

$5.1 N_{f}=2$ in $\mathrm{SU}(3 \mid 1) \mathrm{PQ} \chi \mathrm{PT} \quad 9$

$5.2 \quad N_{f}=2$ plus a quenched strange quark in SU(4|2) PQ $\chi \mathrm{PT} \quad 9$

$\begin{array}{lr}5.3 N_{f}=2+1 \text { in } \mathrm{SU}(4 \mid 1) \mathrm{PQ} \chi \mathrm{PT} & 10\end{array}$

$\begin{array}{lll}5.4 \text { Discussion } & 12\end{array}$

5.5 Applications to lattice QCD 12

6 Conclusions and outlook 13

$\begin{array}{lr}\text { A Propagators in chiral perturbation theory } & 14\end{array}$

$\begin{array}{lll}\text { A.1 Propagators in SU(4|1) chiral perturbation theory } & 14\end{array}$

$\begin{array}{lll}\text { A.2 Propagators in SU(4|2) chiral perturbation theory } & 15\end{array}$

$\begin{array}{ll}\text { B The integral } \bar{B}_{21}\left(q^{2}, M^{2}\right) & 15\end{array}$

\section{Introduction}

Hadronic correlation functions which involve quark disconnected contributions are notoriously hard to compute in lattice QCD $[1,2]$. Such contributions however appear in several quantities related to properties of flavour singlet particles (e.g. the $\eta$ and $\eta^{\prime}$ mesons) or to matrix elements of flavour singlet operators (as for the strangeness content of the nucleon), or to electro-magnetic interactions (e.g. the hadronic contribution to the anomalous magnetic moment of the muon or to the nucleon electric dipole moment). There is no conceptual difficulty in treating disconnected quark diagrams but the computational effort is immense compared to quark-connected contributions. The disconnected part is therefore often neglected, not always providing solid arguments that the systematic uncertainty introduced in this way is under control. 
Here we present a method that allows to predict the quark disconnected contribution to correlation functions in (partially quenched) chiral perturbation theory [3-7]. We first give the general argument and then present an explicit example by deriving predictions for the magnitude of the quark disconnected contribution to the hadronic vacuum polarisation of the photon for $N_{f}=2$ dynamical flavours, for $N_{f}=2$ dynamical flavours with a quenched strange quark and also for the case of $N_{f}=2+1$ active flavours. The vacuum polarisation is the main ingredient in computations of the leading hadronic contribution to the anomalous magnetic moment of the muon and hence of relevance to precision tests of the Standard Model (see ref. [8] for a review of the subject).

We will stay in the Euclidean continuum and infinite volume for most of the discussion. While having applications to lattice QCD in mind, reference to this regularisation is only made where we think that it helps the better understanding of our arguments. We will discuss however the finite volume case in order to show how this technique allows for using partial twisting [9-14] also for form-factors and polarisations involving flavour-diagonal operators.

\section{General argument}

We consider a $n$-point fermionic correlation function in QCD with $N_{f}$ dynamical flavours (not necessarily degenerate). In general several Wick contractions contribute to the correlator. By introducing valence quarks which are degenerate with the dynamical flavours, each Wick contraction can be rewritten in terms of a single fermionic correlation function defined in an un-physical theory. The physical result is recovered by summing over the correlation functions in the un-physical, partially quenched, theory [5-7]. In particular, the approach can be used to separate the contributions from quark disconnected diagrams from those coming from quark connected diagrams. These contributions taken on their own are un-physical, it is therefore natural that in order to define them as correlation functions one has to resort to un-physical theories. Investigations of quark-disconnected diagrams along these lines can also be found in ref. [7].

The number of valence quarks $N_{v}(i)$ degenerate with the $i$ th dynamical flavour, which has to be introduced depends on the particular correlation function. Given a $n$-point fermionic correlation function, $N_{v}(i)$ is related in an obvious way to the largest number $N_{\mathrm{D}}^{\max }(i)$ of disconnected quark loops involving the $i$ th flavour, which can appear as all possible Wick contractions are considered. In particular $N_{v}(i)=N_{\mathrm{D}}^{\max }(i)-1$ at most, in such a way that a different flavour can be attached to each disconnected quark loop. In this approach the partially quenched theory, in which each Wick contraction of the original correlator can be written as an independent correlation function, is not unique but rather depends on the specific $n$-point function we started from.

Partially quenched chiral perturbation theory ( $\mathrm{PQ} \chi \mathrm{PT})$ [5-7], which is an extension of chiral perturbation theory $[3,4]$, provides an asymptotic low energy description of partially quenched QCD (PQQCD) and can therefore be used to obtain predictions for, and algebraic relations amongst, the disconnected and the connected part of a fermionic correlation function. There is a rather large body of literature on PQ $\chi \mathrm{PT}$ and PQQCD, we found the 
reviews in ref. [15] and ref. [16] very clear and useful. In short, following ref. [15], PQQCD can be formulated in terms of a local theory by introducing a commuting spin- $1 / 2$ field, a ghost [17], labelled by $\tilde{q}$ for each valence quark $q$ and by extending the fermionic QCD action by including terms of the form $\bar{q}\left(\not D+m_{q}\right) q+\tilde{q}^{\dagger}\left(\not D+m_{q}\right) \tilde{q}$, which violate the spin statistics theorem. As long as all masses are positive, the determinant produced by the integral over the valence quark fields is cancelled by the corresponding integral over the ghost fields and the QCD partition function is reproduced. The advantage is that this formulation provides field-theoretic expressions for partially quenched correlation functions. As discussed in refs. $[15,16]$, a low energy description of the theory (PQ $\chi \mathrm{PT})$ is obtained by constructing a chiral Lagrangian encoding the apparent chiral symmetry group of the extended action, which is a $S U\left(N_{f}+\sum_{i=1}^{N_{f}} N_{v}(i) \mid \sum_{i=1}^{N_{f}} N_{v}(i)\right)$ graded group.

For the application discussed here the strategy can be exemplified for the case of a meson 2-point function constructed of flavour-diagonal quark-bilinears,

$$
\begin{aligned}
C_{\mathrm{QCD}}(y, x)= & \sum_{q=u, d, s, \ldots}\left\langle\bar{q}(y) \Gamma^{\prime} q(y) \bar{q}(x) \Gamma q(x)\right\rangle_{\mathrm{QCD}} \\
= & -\sum_{q=u, d, s, \ldots}\left\langle\operatorname{Tr}\left\{S_{q}(x, y) \Gamma^{\prime} S_{q}(y, x) \Gamma\right\}\right\rangle_{\mathrm{QCD}} \\
& +\sum_{q=u, d, s, \ldots}\left\langle\operatorname{Tr}\left\{S_{q}(y, y) \Gamma^{\prime}\right\} \operatorname{Tr}\left\{S_{q}(x, x) \Gamma\right\}\right\rangle_{\mathrm{QCD}},
\end{aligned}
$$

where $S_{q}$ is the propagator of the quark field $q$ and $\Gamma^{(\prime)}$ may contain Dirac- as well as colourstructures. The trace is over Dirac- and colour-indices. We will rewrite the correlation function $C_{\mathrm{QCD}}(y, x)$ as the sum of two new correlation functions: one being equivalent to the quark-connected contribution and the other being equivalent to the quark-disconnected contribution. To this end for each quark $q$ we add a valence quark $q_{v}$ which is mass degenerate to it, together with the corresponding ghost field. The correlation $C_{\mathrm{QCD}}(y, x)$ can be rewritten as

$$
\begin{aligned}
C_{\mathrm{QCD}}(y, x)= & \sum_{q=u, d, s, \ldots}\left\langle\bar{q}(y) \Gamma^{\prime} q_{v}(y) \bar{q}_{v}(x) \Gamma q(x)\right\rangle_{\mathrm{PQQCD}} \\
& +\sum_{\substack{q=u, d, s, \ldots \\
\equiv}}\left\langle\bar{q}(y) \Gamma^{\prime} q(y) \bar{q}_{v}^{\prime}(x) \Gamma q_{v}(x)\right\rangle_{\mathrm{PQQCD}} \\
\equiv & C_{\mathrm{PQQCD}}^{\mathrm{Conn}}(y, x)+C_{\mathrm{PQQCD}}^{\mathrm{Disc}}(y, x) .
\end{aligned}
$$

The Wick contractions of the first correlator on the r.h.s. of the first equation lead to quark connected diagrams only, while those for the second correlator produce quark-disconnected diagrams only (see illustrations in figure 1). If we imagine for a moment having regularised the theory on the lattice it is clear that the above equality holds non-perturbatively, since on each gauge configuration the quark propagators and the fermionic determinant (and therefore the weight of the configuration in the path integral) are the same in the two theories. In the following we will show how $C_{\mathrm{PQQCD}}^{\text {Disc }}(y, x)$ and $C_{\mathrm{PQQCD}}^{\mathrm{Conn}}(y, x)$ can be expressed in a suitable chiral effective theory, depending on the flavours entering the sum in eq. (2.1). 


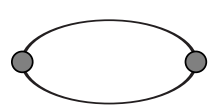

(a)

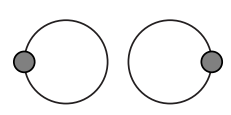

(b)

Figure 1. Wick contractions: (a) connected and (b) disconnected diagram.

\section{Hadronic vacuum polarisation}

The Euclidean hadronic vacuum polarisation (VP) tensor is defined as

$$
\Pi_{\mu \nu}^{\left(N_{f}\right)}(q)=i \int d^{4} x e^{i q x}\left\langle J_{\mu}^{\left(N_{f}\right)}(x) J_{\nu}^{\left(N_{f}\right)}(0)\right\rangle=i C_{\mu \nu}(q),
$$

where $J_{\mu}^{\left(N_{f}\right)}(x)=\sum_{q=1}^{N_{f}} Q_{q} \bar{q}(x) \gamma_{\mu} q(x)$. For $N_{f}=2, q=(u, d)$ and $Q_{q}=(2 / 3,-1 / 3)$ and for $N_{f}=2+1, q=(u, d, s)$ and $Q_{q}=(2 / 3,-1 / 3,-1 / 3)$. Euclidean invariance and current conservation imply

$$
\Pi_{\mu \nu}^{\left(N_{f}\right)}(q)=\left(q_{\mu} q_{\nu}-g_{\mu \nu} q^{2}\right) \Pi^{\left(N_{f}\right)}\left(q^{2}\right) .
$$

For space-like momenta, the relation between $\Pi_{\mu \nu}^{\left(N_{f}\right)}\left(q^{2}\right)$ and the lowest order hadronic contribution $a_{\mu}^{\mathrm{HLO}}$ to the anomalous magnetic moment of the muon has been derived in ref. $[18,19]$ and reads (suppressing the index $N_{f}$ )

$$
a_{\mu}^{\mathrm{HLO}}=\left(\frac{\alpha}{\pi}\right)^{2} \int_{0}^{\infty} d q^{2} f\left(q^{2}\right) \hat{\Pi}\left(q^{2}\right)
$$

with

$$
f\left(q^{2}\right)=\frac{m_{\mu}^{2} q^{2} Z^{3}\left(1-q^{2} Z\right)}{1+m_{\mu}^{2} q^{2} Z^{2}}, \quad Z=-\frac{q^{2}-\sqrt{q^{4}+4 m_{\mu}^{2} q^{2}}}{2 m_{\mu}^{2} q^{2}},
$$

and $\hat{\Pi}\left(q^{2}\right)=4 \pi^{2}\left[\Pi\left(q^{2}\right)-\Pi(0)\right]$. We consider the iso-scalar meson two-point function $C_{\mu \nu}(q)$ in eq. (3.1). The Wick contractions for that correlator lead to quark diagrams of type (a) and (b) as illustrated in figure 1. By considering in some detail the two flavour case we will first separate the disconnected contributions from the connected ones in the way discussed in the previous section and then set-up a computation in the resulting $\mathrm{PQ} \chi \mathrm{PT}$ framework.

According to the discussion above we would need two valence quarks, one degenerate with the $u$ quark and one degenerate with the $d$ quark. However, as we will always assume iso-spin to be an exact symmetry, it is enough to introduce one valence quark, which we call $r$, with the corresponding ghost $r_{\mathrm{g}}$. We therefore have an $\mathrm{SU}(3 \mid 1)$ chiral group. The correlation in eq. (3.1) can then be decomposed as

$$
\begin{aligned}
C_{\mu \nu}(q)=\int d^{4} x e^{i q x} & \left(\frac{4}{9}\left\langle j_{\mu}^{u r}(x) j_{\nu}^{r u}(0)\right\rangle+\frac{1}{9}\left\langle j_{\mu}^{d r}(x) j_{\nu}^{r d}(0)\right\rangle\right. \\
& \left.+\frac{4}{9}\left\langle j_{\mu}^{u u}(x) j_{\nu}^{r r}(0)\right\rangle+\frac{1}{9}\left\langle j_{\mu}^{d d}(x) j_{\nu}^{r r}(0)\right\rangle-\frac{4}{9}\left\langle j_{\mu}^{u u}(x) j_{\nu}^{d d}(0)\right\rangle\right),
\end{aligned}
$$

where $j_{\mu}^{q_{1} q_{2}}(x)=\bar{q}_{1}(x) \gamma_{\mu} q_{2}(x)$. The first two correlators on the r.h.s. are connected, whereas the last three represent the disconnected contributions to the hadronic VP tensor. It is 
convenient to cast the quark-fields into a four-component vector $\psi^{T}=\left(u, d, r, r_{\mathrm{g}}\right)$ and introduce the generators $T^{a}, a=1, \ldots, 15$ of the graded group $\mathrm{SU}(3 \mid 1)$. We use the conventions also employed in [20],

$$
T^{a}=\left(T^{a}\right)^{\dagger}, \quad \operatorname{Str}\left\{T^{a}\right\}=0, \quad \operatorname{Str}\left\{T^{a} T^{b}\right\}=\frac{1}{2} g^{a b}, \quad a=1, \ldots, 15,
$$

with the super-trace $\operatorname{Str}\{A\}=A_{11}+A_{22}+A_{33}-A_{44}$. The matrix $g^{a b}$ reads

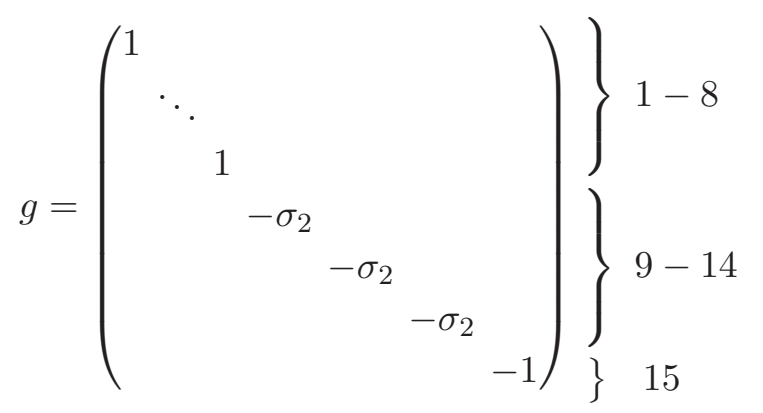

where $\sigma_{2}$ is the second Pauli matrix. $T^{1}, \ldots, T^{8}$ are the generators of the $\mathrm{SU}(3)$ subgroup that acts on the sea and valence components, $T^{9}, \ldots, T^{14}$ mix the quark with the ghost components, $T^{15}$ is the diagonal matrix $\operatorname{diag}(1,1,1,3) /(2 \sqrt{3})$. We also add $T^{0}$ with $\operatorname{Str}\left\{T^{0}\right\}=1 / \sqrt{2}$ which is proportional to the unit matrix in order to describe iso-scalar interactions. For our choice of the generators $T^{0}, \ldots, T^{15}$, the currents in eq. (3.5) can then be rewritten as

$$
\begin{aligned}
& j_{\mu}^{u r}(x)=\bar{\psi}(x) \gamma_{\mu}\left(T^{4}+i T^{5}\right) \psi(x), \\
& j_{\mu}^{r u}(x)=\bar{\psi}(x) \gamma_{\mu}\left(T^{4}-i T^{5}\right) \psi(x), \\
& j_{\mu}^{d r}(x)=\bar{\psi}(x) \gamma_{\mu}\left(T^{6}+i T^{7}\right) \psi(x), \\
& j_{\mu}^{r d}(x)=\bar{\psi}(x) \gamma_{\mu}\left(T^{6}-i T^{7}\right) \psi(x), \\
& j_{\mu}^{u u}(x)=\bar{\psi}(x) \gamma_{\mu}\left(\sqrt{2} T^{0}-\frac{1}{\sqrt{3}} T^{15}+\frac{1}{\sqrt{3}} T^{8}+T^{3}\right) \psi(x), \\
& j_{\mu}^{d d}(x)=\bar{\psi}(x) \gamma_{\mu}\left(\sqrt{2} T^{0}-\frac{1}{\sqrt{3}} T^{15}+\frac{1}{\sqrt{3}} T^{8}-T^{3}\right) \psi(x), \\
& j_{\mu}^{r r}(x)=\bar{\psi}(x) \gamma_{\mu}\left(\sqrt{2} T^{0}-\frac{1}{\sqrt{3}} T^{15}-\frac{2}{\sqrt{3}} T^{8}\right) \psi(x) .
\end{aligned}
$$

This form is more suited for the PQ $\chi \mathrm{PT}$ computation, as it will become clear in the following.

Before concluding this section we note that by using again iso-spin symmetry we could have separated the disconnected and connected parts in the correlator above without introducing any additional valence quark. We have used this completely equivalent approach in ref. [21]. Here however, we preferred to introduce a graded flavour group to provide an example for how one has to proceed for the the more general but also more complicated case of $2+1$ flavours. 


\section{$4 \mathrm{PQ} \chi \mathrm{PT}$ for the connected and disconnected parts of the hadronic VP}

In this section we briefly introduce those parts of the (partially quenched) chiral Lagrangian up to $O\left(p^{4}\right)$ which contribute to the connected as well as to the disconnected piece of the VP $[3,4]$. We took care that the discussion applies to any choice for the (graded) symmetry group. In the next section we will then present results for the cases of SU(3|1), SU(4|2), and $\mathrm{SU}(4 \mid 1)$ flavour groups. These are the relevant symmetry groups for the description of the contributions to the VP in the $N_{f}=2$-theory without and with a quenched strange quark and for the $N_{f}=2+1$-theory, respectively.

\section{1 $O\left(p^{2}\right)$-Lagrangian}

For a generic graded flavour group, the leading order chiral Lagrangian is [3-7]

$$
\mathcal{L}^{(2)}=\frac{F^{2}}{4} \operatorname{Str}\left\{D_{\mu} U D_{\mu} U^{\dagger}\right\}-\frac{1}{2} B F^{2} \operatorname{Str}\left\{M U^{\dagger}+M^{\dagger} U\right\},
$$

where $D_{\mu} U=\partial_{\mu} U+i v_{\mu} U-i U v_{\mu}$ provides the coupling of the meson field to an external vector source $v_{\mu}$ and where

$$
M=\operatorname{diag}\left(m_{1}, \ldots, m_{N_{f}}, m_{1}^{\text {valence }}, \ldots, m_{N}^{\text {valence }}, m_{1}^{\text {ghost }}, \ldots, m_{N}^{\text {ghost }}\right),
$$

contains the dynamical, valence-, and ghost-quark masses. By chirally expanding $U=$ $\exp \left(2 i \frac{\phi^{a} T^{a}}{F}\right)$, where $T^{a}$ are the $\tilde{N}=\left(N_{f}+2 N\right)^{2}-1$ generators of the corresponding $\mathrm{SU}\left(N_{f}+N \mid N\right)$ graded symmetry group (with the generators normalised as in the previous section) and where the $\phi^{a}$ are the Goldstone-boson/fermion fields, we arrive at the $O\left(p^{2}\right)$-expression

$$
\mathcal{L}^{(2)}=\frac{1}{2} g^{a b} \partial_{\mu} \phi^{a} \partial_{\mu} \phi^{b}+2 B \tilde{M}^{a b} \phi^{a} \phi^{b}+\mathcal{L}_{\text {int }}^{(2)},
$$

where $\tilde{M}^{a b}=\operatorname{Str}\left\{M T^{a} T^{b}\right\}$. With $v_{\mu}=v_{\mu}^{a} T^{a}$, the part of the interaction Lagrangian that is of relevance here is

$$
\mathcal{L}_{\mathrm{int}}^{(2)}=\underbrace{-\sum_{k=1}^{\tilde{N}} C_{k}^{b c} g^{a k} \partial_{\mu} \phi^{a} v_{\mu}^{b} \phi^{c}}_{a)} \underbrace{+\frac{1}{2} \sum_{k=1}^{\tilde{N}} \sum_{l=1}^{\tilde{N}} C_{k}^{a b} C_{l}^{c d} g^{k l} v_{\mu}^{a} \phi^{b} \phi^{c} v_{\mu}^{d}}_{b)},
$$

where

$$
C_{a}^{b c}=-2 i \sum_{k=1}^{\tilde{N}} \operatorname{Str}\left\{\left[T^{b}, T^{c}\right] T^{k}\right\} g^{k a},
$$

are the structure constants of the underlying (graded) symmetry group with the (anti-)commutator

$$
\left[T^{a}, T^{b}\right] \equiv T^{a} T^{b}-(-)^{\eta_{a} \eta_{b}} T^{b} T^{a} .
$$

The $\eta_{a}$ (and correspondingly for $\eta_{b}$ ) are 1 if $T^{a}$ mixes valence or sea quarks with a ghost and 0 otherwise (cf. [22]). The Feynman-rules for the vertices illustrated in figure 2 (a) 


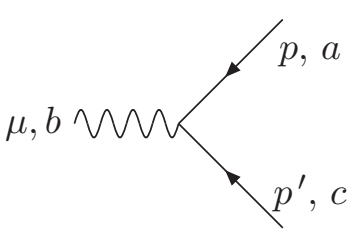

(a)

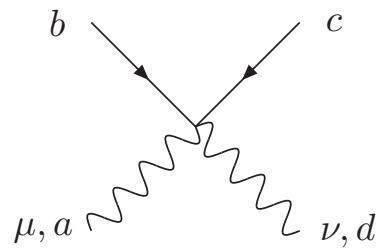

(b)

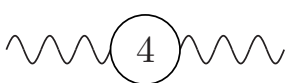

(c)

Figure 2. Contributing vertices in the effective theory.

\begin{tabular}{lcc}
\hline \hline & $X_{1}$ & $X_{2}$ \\
\hline $\mathrm{SU}(2), \mathrm{SU}(3 \mid 1), \mathrm{SU}(4 \mid 2)$ & $-4 h_{2}$ & $h_{s}$ \\
$\mathrm{SU}(3), \mathrm{SU}(4 \mid 1)$ & $L_{10}+2 H_{1}$ & $H_{s}$ \\
\hline
\end{tabular}

Table 1. Gasser-Leutwyler coefficients.

and (b) are then determined as

$$
\begin{array}{r}
-\left(\tilde{C}_{a}^{b c} p_{\mu}+\tilde{C}_{c}^{b a} p_{\mu}^{\prime}\right), \\
\text { b) } i \eta_{\mu \nu} \sum_{k, l=1}^{\tilde{N}} g^{k l}\left(C_{k}^{a b} C_{l}^{c d}+C_{k}^{a c} C_{l}^{b d}\right),
\end{array}
$$

with $\tilde{C}_{a}^{b c}=C_{i}{ }^{b c} g^{a i}$.

\section{2 $O\left(p^{4}\right)$-Lagrangian}

The relevant terms of the $O\left(p^{4}\right)$ Lagrangian $[3,4,23,24]$, i.e. those parts that have a non-zero matrix element between single external vector-sources, are

$$
\mathcal{L}^{(4)}=X_{1} \operatorname{Str}\left\{\hat{v}_{\mu \nu} \hat{v}_{\mu \nu}\right\}+X_{2} \operatorname{Str}\left\{v_{\mu \nu}\right\} \operatorname{Str}\left\{v_{\mu \nu}\right\},
$$

with

$$
v_{\mu \nu}=\partial_{\mu} v_{\nu}-\partial_{\nu} v_{\mu}
$$

and where $\hat{v}_{\mu \nu}$ is the trace-less part $v_{\mu \nu}-\frac{1}{N_{f}} \operatorname{Str}\left\{v_{\mu \nu}\right\}$. As summarised in table 1 the coefficient $X_{1}$ is the shorthand notation for the Gasser-Leutwyler low energy constants of the underlying symmetry group (see for example [3, 4, 25]) and $X_{2}$ parameterises the effective dynamics of flavour-diagonal contributions. The vertices corresponding to these Lagrangians have the following form (cf. figure 2 (c)):

$$
\begin{array}{ll}
\text { c) } i 2 X_{1} g^{a b}\left(\eta_{\mu \nu} p^{2}-p_{\mu} p_{\nu}\right) & \text { for } a, b>0, \\
\text { c) } i 2 X_{2} & \left(\eta_{\mu \nu} p^{2}-p_{\mu} p_{\nu}\right) \text { for } a=b=0 .
\end{array}
$$

\subsection{Contributing diagrams}

At NLO only the diagrams in figure 3 (a) and 3 (b) contribute dynamically to the VP and the diagram in figure 3 (c) provides counter terms. Let $\psi$ be a flavour vector of 


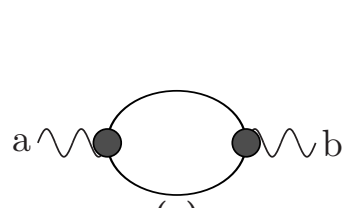

(a)

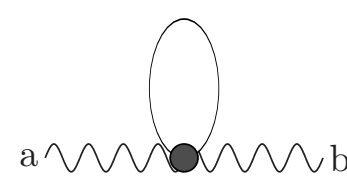

(b)

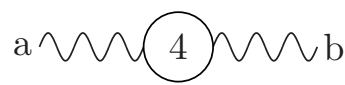

(c)

Figure 3. Feynman diagrams contributing to the hadronic VP at 1-loop level: (a) unitary, (b) tadpole, (c) $O\left(p^{4}\right)$-insertion

$N_{f}$ dynamical quarks, $N$ partially quenched valence quarks and $N$ corresponding ghostquarks. The expression in the effective theory for the Fourier transform of the correlator $i\left\langle\bar{\psi}(x) \gamma_{\mu} T^{a} \psi(x) \bar{\psi}(0) \gamma_{\nu} T^{b} \psi(0)\right\rangle$ at NLO in chiral perturbation theory is of the form

$$
\Pi_{\mu \nu}^{(a, b)}(q, \mu)=\Pi_{\mu \nu}^{(a, b), \text { unit. }}(q)+\Pi_{\mu \nu}^{(a, b), \text { tadp. }}(q)+\Pi_{\mu \nu}^{(a, b), \text { count. }}(\mu)
$$

where $\mu$ is the renormalisation scale. The first term on the r.h.s. corresponds to the unitary diagram,

$$
\begin{aligned}
\Pi_{\mu \nu}^{(a, b), \text { unit. }}(q)= & -\frac{i}{2} \tilde{C}_{i}^{a j} \tilde{C}_{k}^{b l} \\
& \times \int \frac{d^{d} k}{(2 \pi)^{d}}(q+2 k)_{\mu}(q+2 k)_{\nu} G^{i k}\left(k^{2}\right) G^{j l}\left((q-k)^{2}\right),
\end{aligned}
$$

where the overall factor $\frac{1}{2}$ is a symmetry factor. For the second term, the tadpole-diagram (b), one derives

$$
\Pi_{\mu \nu}^{(a, b) \operatorname{tadp} .}=i \eta_{\mu \nu} C_{i}^{a j} \tilde{C}_{i}^{k b} \int \frac{d^{d} k}{(2 \pi)^{d}} G^{j k}\left(k^{2}\right)
$$

For the $\mathrm{SU}(3 \mid 1)$ theory the meson propagators $G^{a b}\left(k^{2}\right)$ can be found e.g. in ref. [20] and we provide the corresponding expressions for $\mathrm{SU}(4 \mid 1)$ and $\mathrm{SU}(4 \mid 2)$ in appendix A. The last term, $\Pi_{\mu \nu}^{(a, b), \text { count. }}(\mu)$, contains the counter terms. We compute $\Pi_{\mu \nu}^{(a, b)}(q, \mu)$ in dimensional regularisation as explained in detail in ref. [26] where also the solutions for the loop integrals have been derived and reduced to the integral $\bar{B}_{21}\left(q^{2}, M^{2}\right)$ defined in appendix B.

\section{$5 \quad$ Results and applications to lattice QCD}

Here we present the results for the VP at NLO in chiral perturbation theory for various underlying graded flavour-symmetry groups. In particular, we consider the theories with $N_{f}=2$ flavours without or with an additional quenched strange quark and also the $N_{f}=$ $2+1$ theory. In each case we present the expressions in the effective theory for the full VP and also for the contributions from quark-connected and quark-disconnected diagrams. 


\section{$\mathbf{5 . 1} \quad N_{f}=2$ in $\mathrm{SU}(3 \mid 1) \mathbf{P Q} \chi \mathbf{P T}$}

For the theory with two dynamical and degenerate light quarks only we found the following expressions for the VP:

$$
\begin{aligned}
& \Pi_{\text {Full }}^{(3 \mid 1)}\left(q^{2}\right)=-\left(\Lambda^{(3 \mid 1)}(\mu)+\frac{2}{9} h_{s}+i 4 \bar{B}_{21}\left(\mu^{2}, q^{2}, M_{\pi}^{2}\right)\right), \\
& \Pi_{\text {Conn }}^{(3 \mid 1)}\left(q^{2}\right)=-\frac{10}{9}\left(\Lambda^{(3 \mid 1)}(\mu)+i 4 \bar{B}_{21}\left(\mu^{2}, q^{2}, M_{\pi}^{2}\right)\right), \\
& \Pi_{\text {Disc }}^{(3 \mid 1)}\left(q^{2}\right)=\frac{1}{9}\left(\Lambda^{(3 \mid 1)}(\mu)-2 h_{s}+i 4 \bar{B}_{21}\left(\mu^{2}, q^{2}, M_{\pi}^{2}\right)\right),
\end{aligned}
$$

where

$$
\Lambda^{(3 \mid 1)}(\mu)=-8 h_{2}(\mu)
$$

The integral $\bar{B}_{21}\left(\mu^{2}, q^{2}, M^{2}\right)$ is defined in appendix B. All contributions are parameterised in terms of the low-energy constant $h_{2}$ and the pion mass $M_{\pi}$. The full expression as well as the disconnected piece also depend on the parameter $h_{s}$. This is a peculiarity of the twoflavour theory and we will see in the next sub-sections that the corresponding expressions in the presence of a strange valence quark do not depend on this additional parameter. Hence, a prediction of the quark-disconnected diagram is not possible without the knowledge of $h_{2}$ and $h_{s}$. These parameters however, can in principle be determined from simulations of lattice QCD and $h_{s}$ can also be obtained by matching to the expressions in the next section.

The application we have in mind when discussing the VP is the leading hadronic contribution to the muon anomalous magnetic moment defined in eq. (3.3). As discussed in section $3, a_{\mu}^{\mathrm{HLO}}$ depends on $\hat{\Pi}\left(q^{2}\right)=4 \pi^{2}\left[\Pi\left(q^{2}\right)-\Pi(0)\right]$. We observe that all reference to the low-energy constants disappears in this difference at NLO in the effective theory and we find [21]

$$
\frac{\hat{\Pi}_{\text {Disc }}\left(q^{2}\right)}{\hat{\Pi}_{\text {Conn }}\left(q^{2}\right)}=-\frac{1}{10}
$$

At this order in the effective theory it is therefore sufficient to compute the quark-connected piece contributing to $a_{\mu}^{\mathrm{HLO}}$ and then correct for the quark-disconnected piece using the above relation which predicts a $10 \%$ negative shift for all values of the momentum (note in this context the discussion in section 5.4). The disconnected contribution at NLO turns out to have the same momentum and quark-mass dependence as the connected piece.

\section{2 $N_{f}=2$ plus a quenched strange quark in $\mathrm{SU}(4 \mid 2) \mathbf{P Q} \chi \mathbf{P T}$}

For the theory with two dynamical and degenerate light quarks and one quenched strange quark one needs to consider SU(4|2) PQ $\chi$ PT, where we found the following expressions for 
the vacuum polarisation:

$$
\begin{aligned}
& \Pi_{\text {Full }}^{(4 \mid 2)}\left(q^{2}\right)=\Lambda^{(4 \mid 2)}(\mu)-4 i\left(\quad \bar{B}_{21}\left(\mu^{2}, q^{2}, M_{\pi}^{2}\right)-\frac{1}{9} \bar{B}_{21}\left(\mu^{2}, q^{2}, M_{s s}^{2}\right)+\frac{4}{9} \bar{B}_{21}\left(\mu^{2}, q^{2}, M_{K}^{2}\right)\right), \\
& \Pi_{\mathrm{Conn}}^{(4 \mid 2)}\left(q^{2}\right)=\Lambda^{(4 \mid 2)}(\mu)-4 i\left(\frac{10}{9} \bar{B}_{21}\left(\mu^{2}, q^{2}, M_{\pi}^{2}\right) \quad+\frac{2}{9} \bar{B}_{21}\left(\mu^{2}, q^{2}, M_{K}^{2}\right)\right), \\
& \Pi_{\text {Disc }}^{(4 \mid 2)}\left(q^{2}\right)=\quad-4 i\left(-\frac{1}{9} \bar{B}_{21}\left(\mu^{2}, q^{2}, M_{\pi}^{2}\right)-\frac{1}{9} \bar{B}_{21}\left(\mu^{2}, q^{2}, M_{s s}^{2}\right)+\frac{2}{9} \bar{B}_{21}\left(\mu^{2}, q^{2}, M_{K}^{2}\right)\right),
\end{aligned}
$$

where

$$
\Lambda^{(4 \mid 2)}(\mu)=8 h_{2}(\mu) .
$$

The full expression and the connected piece depend on the low energy constant $h_{2}$ and the pion and kaon mass. An additional dependence on the mass of the strange-quark enters in terms of the mass $M_{s s}^{2}=2 B m_{s}$ of an un-physical meson made by the two quenched strange quarks. It is well known that in the $N_{f}=2+1$-theory the quark-disconnected contribution to the hadronic VP vanishes in the limit of equal quark-masses. This SU(3) symmetry prohibits the presence of low-energy constants in the expression for the quark-disconnected diagrams at NLO and this is indeed what we found when computing $\Pi_{\text {Disc }}^{(4 \mid 2)}\left(q^{2}\right)$. At $\mathrm{NLO}$ in the effective theory we therefore provide an entirely parameter-free prediction of the quark-disconnected diagram. Moreover, as in the case of the $N_{f}=2$-theory, also $\hat{\Pi}\left(q^{2}\right)$ is free of low-energy constants and a parameter-free prediction for the ratio of the quark-disconnected contribution to the quark-connected contribution can be made. As can be seen in figure 4 (a), in the limits $M_{K} \rightarrow \infty$ and $M_{K}=M_{\pi}$, the above formulae reproduce the results for the $N_{f}=2$ theory and the vanishing of the disconnected piece in the $M_{K}=M_{\pi}$-limit, respectively.

\section{3 $N_{f}=2+1$ in $\mathrm{SU}(4 \mid 1) \mathbf{P Q} \chi \mathbf{P T}$}

For the theory with two dynamical and degenerate light quarks and one dynamical strange quark we found the following expressions for the vacuum polarisation:

$$
\begin{aligned}
& \Pi_{\text {Full }}^{(4 \mid 1)}\left(q^{2}\right)=\Lambda^{(4 \mid 1)}(\mu)-4 i\left(\bar{B}_{21}\left(\mu^{2}, q^{2}, M_{\pi}^{2}\right)+\bar{B}_{21}\left(\mu^{2}, q^{2}, M_{K}^{2}\right)\right), \\
& \Pi_{\text {Conn }}^{(4 \mid 1)}\left(q^{2}\right)=\Lambda^{(4 \mid 1)}(\mu)-4 i\left(\frac{10}{9} \bar{B}_{21}\left(\mu^{2}, q^{2}, M_{\pi}^{2}\right)+\frac{1}{9} \bar{B}_{21}\left(\mu^{2}, q^{2}, M_{s s}^{2}\right)+\frac{7}{9} \bar{B}_{21}\left(\mu^{2}, q^{2}, M_{K}^{2}\right)\right), \\
& \Pi_{\text {Disc }}^{(4 \mid 1)}\left(q^{2}\right)=\quad-4 i\left(-\frac{1}{9} \bar{B}_{21}\left(\mu^{2}, q^{2}, M_{\pi}^{2}\right)-\frac{1}{9} \bar{B}_{21}\left(\mu^{2}, q^{2}, M_{s s}^{2}\right)+\frac{2}{9} \bar{B}_{21}\left(\mu^{2}, q^{2}, M_{K}^{2}\right)\right),
\end{aligned}
$$

where

$$
\Lambda^{(4 \mid 1)}=-2\left(L_{10}(\mu)+2 H_{1}(\mu)\right) .
$$

As in the previous sub-section, the full expression and the connected piece depend on low-energy constants, this time $L_{10}$ and $H_{1}$, and the pion and kaon mass. Also here a dependence on the mass of the strange-quark enters in terms of the mass $M_{s s}^{2}=2 B m_{s}$ of an un-physical meson made of a dynamical and a quenched strange quark. In the 


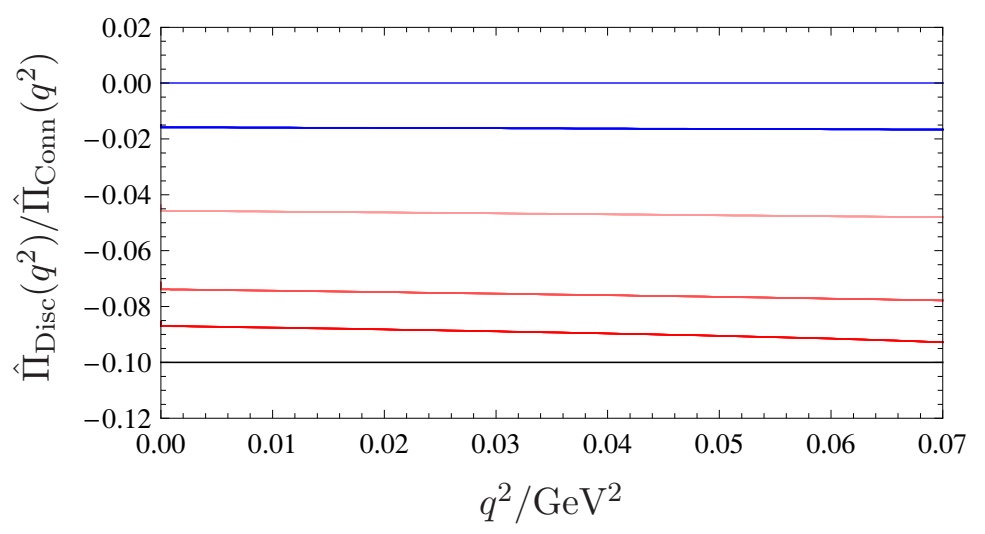

(a)

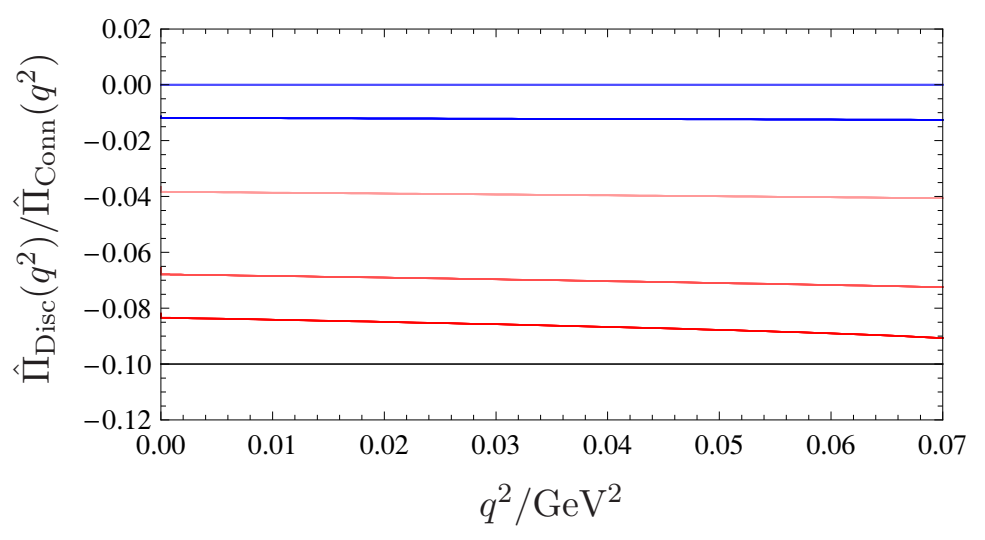

(b)

Figure 4. The plots illustrate the ratio of the contributions from quark-disconnected and quarkconnected diagrams to the VP as a function of the momentum $q^{2}$ at NLO in chiral perturbation theory for the cases (a) $N_{f}=2$ with a quenched strange quark, (b) $N_{f}=2+1$. In both cases the lines are from top to bottom: the limit $M_{\pi}=M_{K}$, then fixed $M_{K}=495 \mathrm{MeV}$ and $M_{\pi}=$ $400,300,200,139 \mathrm{MeV}$. The bottom most-line at $-1 / 10$ is the result for the $N_{f}=2$ theory.

same way as in the previous section $\mathrm{SU}(3)$ symmetry prohibits the presence of low-energy constants in the expression for the quark-disconnected diagram at NLO. At this order in the effective theory we are therefore able to provide an entirely parameter-free prediction of the quark-disconnected diagram. Again, $\hat{\Pi}\left(q^{2}\right)$ is free of low-energy constants and a parameter-free prediction for the ratio of the quark-disconnected contribution to the quark-connected contribution can be made. We illustrate this result in figure 4 (b). In the same way as for the $N_{f}=2$ theory with a quenched strange quark, in the limits limits $M_{K} \rightarrow \infty$ and $M_{K}=M_{\pi}$, the above formulae reproduce the results for the $N_{f}=2$ theory and the vanishing of the disconnected piece in the $\mathrm{SU}(3)$-limit, respectively. As a further check, our formulae reproduce the result in ref. [26]. From the comparison of the plots (a) and (b) we see little influence of the dynamical strange quark. 


\subsection{Discussion}

Before we suggest applications to simulations of lattice QCD we would like to point out obvious limitations of the above formulae. While the underlying idea of the approach, i.e. eq. (2.2), is exact in QCD and in the chiral effective theory, the results presented here are only valid up to NLO chiral perturbation theory. If at all, this approximation only holds for small quark-masses and only for small values of the momentum. There are in fact doubts that the physical strange quark mass can be described reliably within chiral perturbation theory [27]. One might hope that cancellations in the ratio $\hat{\Pi}_{\text {Disc }}\left(q^{2}\right) / \hat{\Pi}_{\text {Conn }}\left(q^{2}\right)$ will improve the convergence of the expansion in the meson masses and the momentum. However, only expensive numerical simulations in lattice QCD and higher order computations in the effective theory will be able to tell.

At least as important is the fact that instead of coupling to two pions, the photon will also couple to vector resonances. It is a priori not clear if at all or in which kinematical regime chiral perturbation theory parameterises these dynamics correctly in terms of the low-energy constants of the chiral effective Lagrangian. Aubin and Blum [28] for example found indications for vector-dominance in the analysis of their lattice QCD data for the hadronic VP. We expect sizeable corrections to the expressions found here. There do exist models, generally known as "resonace $\chi \mathrm{PT}$ " models, where the vector resonances are dynamical degrees of freedom (see e.g. [29]). Conceptually these models are not as solid as $\chi \mathrm{PT}$, to the extent that no systematic power counting can be formulated. While clearly desirable, a study of how the vector degrees of freedom will modify the expressions is beyond the scope of the current work but in principle technically straight forward within the framework of resonace $\chi \mathrm{PT}$, which is however subject to the theoretical issues mentioned above.

Given these remarks, the results presented here can only provide an order-of-magnitude estimate and should be used with great care. In the absence of any other quantitative knowledge of the quark-disconnected diagrams, the results can be used in order to correct the lattice data for the connected contributions.

\subsection{Applications to lattice QCD}

Partially twisted boundary conditions for the VP: Partially twisted boundary conditions [9-14] by now have become a standard tool in lattice hadron phenomenology. For the boundary condition $q_{i}\left(x_{k}+L\right)=e^{i \theta_{k, i}} q_{i}(x)$ for the valence quark-flavours $q_{1}$ and $q_{2}$ of a pseudo-scalar meson of mass $m$ in a finite lattice of spatial extent $L$, the dispersion relation takes the form $E\left(\theta_{1}, \theta_{2}\right)=\sqrt{m^{2}+\left(\theta_{1} / L-\theta_{2} / L\right)^{2}}[9,12,14]$, which shows how the periodicity of the fermionic fields can be modified in order to induce spacial momentum to hadrons. Besides an exponentially suppressed and computable finite size effect the choice of quark-field boundary conditions for processes with only one initial and/or final hadron state introduces no further systematics [9]. The effect of partial twisting cancels however in flavour-neutral mesons, as is clear from the form of the above dispersion relation. Due to the flavour-diagonal structure of the electro-magnetic current, partial twisting would therefore naively have no effect on the hadronic VP. The prescription introduced here however, allows for isolating a flavour-off-diagonal connected contribution and thus for inducing ar- 
bitrary values of the momentum for the VP. In other words we can assign different twisting angles to the quarks $q$ and $q_{v}$ in eq. (2.2), which has the net effect of inducing momentum in the connected part. The effectiveness of this method in the numerical computation of the connected part of the hadronic VP will be illustrated in a forthcoming publication $[30,31]$. The relations in the chiral effective theory between the quark-connected and the quark-disconnected piece then allow to predict also the momentum-dependence of the disconnected piece. A very similar argument was already used in refs. [32, 33] in order to justify the use of partially twisted boundary conditions for the pion's vector form factor. The exponential suppression in the volume of the isospin breaking introduced by partially twisting only one of the light quarks is also discussed in refs. [32, 33].

\section{Conclusions and outlook}

In this paper we show how quark-disconnected contributions to hadronic $n$-point functions can be estimated within the framework of partially quenched chiral perturbation theory. As an example we derive predictions for the quark-disconnected contribution to the photon's vacuum polarisation for QCD with two degenerate light quarks, with and without a quenched strange quark, and also for the case of the three-flavour theory. In the presence of a quenched strange quark the prediction for the quark-disconnected contribution turns out to be parameter-free.

The vacuum polarisation is currently computed by various lattice collaborations with the aim to make first-principles predictions for the leading hadronic contribution to the muon anomalous moment $[21,28,34]$. Since precise computations of quark-disconnected correlators from first principles still remain numerically extremely expensive they are often neglected in such computations. The approach presented here will allow to improve estimates of the systematic error introduced in this way.

The method can be applied to other physical observables of interest that receive contributions from quark-disconnected diagrams, like the strange-quark content in the nucleon, the nucleon electric dipole moment or the pion scalar form-factor. The discussion presented here is limited to NLO chiral perturbation theory. As a next step we plan to include the vector degrees of freedom and it would also be important to extend the computation for the case of the vacuum polarisation to higher orders in the chiral expansion in order to assess how robust the predictions reported here are. The inclusion of the leading lattice artifacts in the effective theory is also straight forward within the framework set up by Sharpe and Singleton in ref. [35].

A spin-off of the ideas presented here is a method that allows for projecting (the connected part of) correlators containing flavour-diagonal currents onto any desired momentum using partially twisted boundary conditions.

\section{Acknowledgments}

We thank Dalibor Djukanovic, Silvia Necco, Hartmut Wittig, and Steve Sharpe for illuminating discussions. 


\section{A Propagators in chiral perturbation theory}

Here we provide the definition of propagators in $\mathrm{PQ} \chi \mathrm{PT}$ with the graded symmetry groups $\mathrm{SU}(4 \mid 1)$ and $\mathrm{SU}(4 \mid 2)$. For simplicity all results are quoted in the limit where the Goldstone bosons and the corresponding ghosts are mass-degenerate. This limit is sufficient for the discussion in the main body of this paper. For completeness we note that the case $\mathrm{SU}(3 \mid 1)$ has been worked out nicely in [20] and for most of the discussion we adopt the conventions laid out there.

\section{A.1 Propagators in SU(4|1) chiral perturbation theory}

We consider the case of the effective theory with $N_{f}=2+1$ dynamical flavours with two degenerate light quarks of mass $m$, one strange quark of mass $m_{s}$ and one quenched $r$ quark with $m_{s}=m_{r}=m_{r_{g}}$. The dynamical (not necessarily physical) degrees of freedom in the effective theory have masses

$$
M_{\pi}^{2}=2 B m, \quad M_{K}^{2}=B\left(m+m_{s}\right), \quad M_{s s}^{2}=2 B m_{s} .
$$

The propagator $G^{i j}\left(k^{2}\right)$ is defined as follows: The Lagrangian in eq. (4.3) with $\mathcal{L}_{\text {int }}^{(2)}=0$ can be rewritten as

$$
\mathcal{L}^{(2)}=\frac{1}{2} g^{a b}\left(\partial_{\mu} \phi^{a} \partial_{\mu} \phi^{b}+M_{a}^{2} \phi^{a} \phi^{b}\right)+\frac{1}{6} h^{a b}\left(M_{\pi}^{2}-M_{K}^{2}\right) \phi^{a} \phi^{b},
$$

where

$$
M_{a}^{2}= \begin{cases}M_{\pi}^{2} & \text { for } a=1,2,3, \\ \frac{1}{2}\left(M_{\pi}^{2}+M_{K}^{2}\right) & \text { for } a=4,5,6,7,9,10,11,12,16,17,18,19, \\ M_{s s}^{2} & \text { for } a=8,13,14,15,20,21,22,23,24\end{cases}
$$

and where for our choice of the $\mathrm{SU}(4 \mid 1)$-generators

$$
\begin{aligned}
h^{a b} & =\left(\sqrt{2} g^{a 8}+g^{a 15}-g^{a 24}\right)\left(\sqrt{2} g^{b 8}+g^{b 15}-g^{b 24}\right), \\
l^{a b} & =-\left(\sqrt{2} g^{a 8}+g^{a 15}+g^{a 24}\right)\left(\sqrt{2} g^{b 8}+g^{b 15}+g^{b 24}\right) .
\end{aligned}
$$

In terms of these matrices the propagator is given by

$$
G^{a b}\left(k^{2}\right)=g^{a b} G_{1}\left(k^{2}, M_{a}^{2}\right)+\frac{1}{3} l^{a b}\left(M_{\pi}^{2}-M_{K}^{2}\right) G_{2}\left(k^{2}, M_{\eta}^{2}, M_{s s}^{2}\right),
$$

where we assume $M_{\eta}^{2}=2 B\left(2 m_{s}+m\right) / 3$ and where

$$
\begin{aligned}
G_{1}\left(k^{2}, M^{2}\right) & =\frac{1}{\left(k^{2}+M^{2}\right)}, \\
G_{2}\left(k^{2}, M_{1}^{2}, M_{2}^{2}\right) & =\frac{1}{\left(k^{2}+M_{1}^{2}\right)\left(k^{2}+M_{2}^{2}\right)} .
\end{aligned}
$$




\section{A.2 Propagators in $\mathrm{SU}(4 \mid 2)$ chiral perturbation theory}

We consider the case of the effective theory with $N_{f}=2$ dynamical flavours with two degenerate light quarks of mass $m$ and two quenched quarks $s$ and $r$ with the mass of the strange quark $m_{s}=m_{s_{g}}=m_{r}=m_{r_{g}}$. The dynamical degrees of freedom in the effective theory have masses

$$
M_{\pi}^{2}=2 B m, \quad M_{K}^{2}=B\left(m+m_{s}\right), \quad M_{s s}^{2}=2 B m_{s} .
$$

The propagator $G^{a b}\left(k^{2}\right)$ is defined as follows: The Lagrangian in eq. (4.3) restricted to lowest order and with $\mathcal{L}_{\text {int }}^{(2)}=0$ can be rewritten as

$$
\mathcal{L}^{(2)}=\frac{1}{2} g^{a b}\left(\partial_{\mu} \phi^{a} \partial_{\mu} \phi^{b}+M_{a}^{2} \phi^{a} \phi^{b}\right)+\frac{1}{6} h^{a b}\left(M_{\pi}^{2}-M_{K}^{2}\right) \phi^{a} \phi^{b},
$$

where

$$
M_{a}^{2}= \begin{cases}M_{\pi}^{2} & \text { for } a=1,2,3, \\ \frac{1}{2}\left(M_{\pi}^{2}+M_{K}^{2}\right) & \text { for } a=4,5,6,7,9,10,11,12,16,17,18,19,25,26,27,28, \\ M_{s s}^{2} & \text { for } a=8,13,14,15,20,21,22,23,24,29,30,31,32,33,34,35,\end{cases}
$$

and where for our choice of the $\mathrm{SU}(4 \mid 2)$-generators

$$
\begin{aligned}
h^{a b} & =\left(\sqrt{2} g^{a 8}+g^{a 15}-g^{a 24}-\sqrt{2} g^{a 35}\right)\left(\sqrt{2} g^{b 8}+g^{b 15}-g^{b 24}-\sqrt{2} g^{b 35}\right), \\
l^{a b} & =-\left(\sqrt{2} g^{a 8}+g^{a 15}+g^{a 24}+\sqrt{2} g^{a 35}\right)\left(\sqrt{2} g^{b 8}+g^{b 15}+g^{b 24}+\sqrt{2} g^{b 35}\right) .
\end{aligned}
$$

In terms of these matrices the propagator is given by

$$
G^{a b}\left(k^{2}\right)=g^{a b} G_{1}\left(k^{2}, M_{a}^{2}\right)+\frac{1}{3} l^{a b}\left(M_{\pi}^{2}-M_{K}^{2}\right) G_{2}\left(k^{2}, M_{s s}^{2}, M_{s s}^{2}\right),
$$

where $G_{1}$ and $G_{2}$ are defined as in eq. (A.6).

\section{B The integral $\bar{B}_{21}\left(q^{2}, M^{2}\right)$}

Here we just quote the integral itself. Its derivation from the sum of the unitary contribution in eq. (4.12) and the tadpole in eq. (4.13) can be found for example in the appendix of ref. [26]:

$$
\bar{B}_{21}\left(q^{2}, M^{2}\right)=\frac{1}{12}\left[\left(1-\frac{4 M^{2}}{q^{2}}\right) \bar{B}\left(q^{2}, M^{2}\right)-\frac{i}{48 \pi^{2}}\right]
$$

where for $q^{2}<4 M^{2}$

$$
\begin{aligned}
\bar{B}\left(q^{2}, M^{2}\right) & =-\frac{i}{16 \pi^{2}} \int_{0}^{1} d x \log \left(1-x(1-x) \frac{q^{2}}{M^{2}}\right) \\
x & =\frac{\frac{4 M^{2}}{q^{2}}}{=}-\frac{i}{16 \pi^{2}}\left[-2+\sqrt{1-x} \log \left(\frac{\sqrt{1-x}+1}{\sqrt{1-x}-1}\right)\right] \\
& =\frac{i}{96 \pi^{2}} \frac{q^{2}}{M^{2}}+\frac{i}{960 \pi^{2}} \frac{q^{4}}{M^{4}}+\ldots
\end{aligned}
$$


In the body of this paper we found it convenient to absorb a logarithmic term into the expression for $\bar{B}_{21}$ :

$$
\bar{B}_{21}\left(\mu^{2}, p^{2}, M^{2}\right)=\bar{B}_{21}\left(p^{2}, M^{2}\right)-\frac{i}{4} \frac{1}{48 \pi^{2}} \log \left(\frac{M^{2}}{\mu^{2}}\right) .
$$

Open Access. This article is distributed under the terms of the Creative Commons Attribution Noncommercial License which permits any noncommercial use, distribution, and reproduction in any medium, provided the original author(s) and source are credited.

\section{References}

[1] H. Neff, N. Eicker, T. Lippert, J.W. Negele and K. Schilling, On the low fermionic eigenmode dominance in QCD on the lattice, Phys. Rev. D 64 (2001) 114509 [hep-lat/0106016] [SPIRES].

[2] J. Foley et al., Practical all-to-all propagators for lattice $Q C D$, Comput. Phys. Commun. 172 (2005) 145 [hep-lat/0505023] [SPIRES].

[3] J. Gasser and H. Leutwyler, Chiral perturbation theory to one loop, Ann. Phys. 158 (1984) 142 [SPIRES].

[4] J. Gasser and H. Leutwyler, Chiral perturbation theory: expansions in the mass of the strange quark, Nucl. Phys. B 250 (1985) 465 [SPIRES].

[5] C.W. Bernard and M.F.L. Golterman, Chiral perturbation theory for the quenched approximation of QCD, Phys. Rev. D 46 (1992) 853 [hep-lat/9204007] [SPIRES].

[6] C.W. Bernard and M.F.L. Golterman, Partially quenched gauge theories and an application to staggered fermions, Phys. Rev. D 49 (1994) 486 [hep-lat/9306005] [SPIRES].

[7] S.R. Sharpe and N. Shoresh, Physical results from unphysical simulations, Phys. Rev. D 62 (2000) 094503 [hep-lat/0006017] [SPIRES].

[8] F. Jegerlehner and A. Nyffeler, The muon g-2, Phys. Rept. 477 (2009) 1 [arXiv:0902.3360] [SPIRES].

[9] C.T. Sachrajda and G. Villadoro, Twisted boundary conditions in lattice simulations, Phys. Lett. B 609 (2005) 73 [hep-lat/0411033] [SPIRES].

[10] P.F. Bedaque and J.-W. Chen, Twisted valence quarks and hadron interactions on the lattice, Phys. Lett. B 616 (2005) 208 [hep-lat/0412023] [SPIRES].

[11] P.F. Bedaque, Aharonov-Bohm effect and nucleon nucleon phase shifts on the lattice, Phys. Lett. B 593 (2004) 82 [nucl-th/0402051] [SPIRES].

[12] G.M. de Divitiis, R. Petronzio and N. Tantalo, On the discretization of physical momenta in lattice QCD, Phys. Lett. B 595 (2004) 408 [hep-lat/0405002] [SPIRES].

[13] B.C. Tiburzi, Twisted quarks and the nucleon axial current, Phys. Lett. B 617 (2005) 40 [hep-lat/0504002] [SPIRES].

[14] UKQCD collaboration, J.M. Flynn, A. Jüttner and C.T. Sachrajda, A numerical study of partially twisted boundary conditions, Phys. Lett. B 632 (2006) 313 [hep-lat/0506016] [SPIRES].

[15] S.R. Sharpe, Applications of chiral perturbation theory to lattice QCD, hep-lat/0607016 [SPIRES]. 
[16] M. Golterman, Applications of chiral perturbation theory to lattice QCD, arXiv:0912.4042 [SPIRES].

[17] A. Morel, Chiral logarithms in quenched QCD, J. Phys. (France) 48 (1987) 1111 [SPIRES].

[18] T. Blum, Lattice calculation of the lowest order hadronic contribution to the muon anomalous magnetic moment, Phys. Rev. Lett. 91 (2003) 052001 [hep-lat/0212018] [SPIRES].

[19] QCDSF collaboration, M. Göckeler et al., Vacuum polarisation and hadronic contribution to muon g-2 from lattice QCD, Nucl. Phys. B 688 (2004) 135 [hep-lat/0312032] [SPIRES].

[20] L. Giusti and M. Lüscher, Chiral symmetry breaking and the Banks-Casher relation in lattice QCD with Wilson quarks, JHEP 03 (2009) 013 [arXiv:0812.3638] [SPIRES].

[21] A. Jüttner and M. Della Morte, New ideas for g-2 on the lattice, PoS (LAT2009) 143 [arXiv:0910.3755] [SPIRES].

[22] S. Weinberg, The quantum theory of fields, volume 3: supersymmetry, Cambridge University Press, Cambridge U.K. (2005) [SPIRES].

[23] R. Kaiser, Anomalies and WZW-term of two-flavour QCD, Phys. Rev. D 63 (2001) 076010 [hep-ph/0011377] [SPIRES].

[24] R. Kaiser and H. Leutwyler, Large- $N_{c}$ in chiral perturbation theory, Eur. Phys. J. C 17 (2000) 623 [hep-ph/0007101] [SPIRES].

[25] J. Bijnens, G. Colangelo and G. Ecker, Renormalization of chiral perturbation theory to order $p^{6}$, Ann. Phys. 280 (2000) 100 [hep-ph/9907333] [SPIRES].

[26] E. Golowich and J. Kambor, Two loop analysis of vector current propagators in chiral perturbation theory, Nucl. Phys. B 447 (1995) 373 [hep-ph/9501318] [SPIRES].

[27] RBC-UKQCD collaboration, C. Allton et al., Physical results from $2+1$ flavor domain wall $Q C D$ and $\mathrm{SU}(2)$ chiral perturbation theory, Phys. Rev. D 78 (2008) 114509 [arXiv: 0804.0473] [SPIRES].

[28] C. Aubin and T. Blum, Calculating the hadronic vacuum polarization and leading hadronic contribution to the muon anomalous magnetic moment with improved staggered quarks, Phys. Rev. D 75 (2007) 114502 [hep-lat/0608011] [SPIRES].

[29] G. Ecker, J. Gasser, A. Pich and E. de Rafael, The role of resonances in chiral perturbation theory, Nucl. Phys. B 321 (1989) 311 [SPIRES].

[30] B.B. Brandt et al., Wilson fermions at fine lattice spacings: scale setting, pion form factors and $(g-2)_{\mu}$, arXiv: 1010.2390 [SPIRES].

[31] M. Della Morte, B. Jäger, A. Jüttner and H. Wittig, in preparation.

[32] P.A. Boyle, J.M. Flynn, A. Jüttner, C.T. Sachrajda and J.M. Zanotti, Hadronic form factors in lattice QCD at small and vanishing momentum transfer, JHEP 05 (2007) 016 [hep-lat/0703005] [SPIRES].

[33] F.-J. Jiang and B.C. Tiburzi, Flavor twisted boundary conditions in the Breit frame, Phys. Rev. D 78 (2008) 037501 [arXiv:0806.4371] [SPIRES].

[34] D.B. Renner and X. Feng, Hadronic contribution to g-2 from twisted mass fermions, PoS (LATTICE 2008) 129 [arXiv: 0902.2796] [SPIRES].

[35] S.R. Sharpe and R.L. Singleton Jr, Spontaneous flavor and parity breaking with Wilson fermions, Phys. Rev. D 58 (1998) 074501 [hep-lat/9804028] [SPIRES]. 